\title{
High-grain diets supplemented with phytogenic compounds or autolyzed yeast modulate ruminal bacterial community and fermentation in dry cows
}

\author{
V. Neubauer, ${ }^{\star} \dagger$ R. Petri, ${ }^{*}$ E. Humer, ${ }^{*}$ I. Kröger, ${ }^{*}$ E. Mann, $†$ N. Reisinger,‡ M. Wagner,† and Q. Zebeli*1 \\ *Institute of Animal Nutrition and Functional Plant Compounds, and \\ †Institute for Milk Hygiene, Milk Technology and Food Science, Department for Farm Animals and Veterinary Public Health, \\ University of Veterinary Medicine, Veterinaerplatz 1, 1210 Vienna, Austria \\ ‡BIOMIN Research Center, BIOMIN Holding GmbH, 3430 Tulln, Austria
}

\begin{abstract}
The feeding of concentrate-rich diets may lead to microbial imbalances and dysfermentation in the rumen. The main objective of this study was to determine the effects of supplementing phytogenic compounds (PHY) or autolyzed yeast (AY) on rumen fermentation and microbial abundance in cows intermittently fed concentrate-rich diets. The experiment was carried out as an incomplete $3 \times 4$ Latin square design, with 8 nonlactating rumen-fistulated Holstein-Friesian cows. The cows were randomly assigned to a concentrate diet that was either not supplemented (CON), or supplemented with PHY or AY. Each of the 4 consecutive experimental periods was composed of a 1-wk roughage-only diet (RD), 6-d gradual concentrate increase, followed by 1 wk of $65 \%$ concentrate (dry matter basis; Conc I), and 1 wk of RD and a final 2-wk $65 \%$ concentrate (dry matter basis; Conc II) phase. Digesta samples were collected from the rumen mat for bacterial 16S rRNA gene Illumina MiSeq (Illumina, Balgach, Switzerland) sequencing, and samples of particle-associated rumen liquid were obtained for measuring short-chain fatty acids, lactate, ammonia, and pH during RD (d 6), Conc I (d 19), and Conc II (d 39). The concentrate feeding caused a decrease of overall bacterial diversity indices, especially during Conc I. The genera Ruminococcus, Butyrivibrio, and Coprococcus were decreased, whereas Prevotella, Megasphaera, Lachnospira, and Bacteroides were increased in abundance. Supplementation of both feed additives increased the abundance of gram-positive and decreased that of gram-negative bacteria. Supplementation of AY enhanced cellulolytic bacteria such as Ruminococcus spp., whereas PHY decreased starch and sugar fermenters including Bacteroides spp., Shuttleworthia spp., and Syntrophococcus spp. Moreover, PHY supplementation increased butyrate percentage in the
\end{abstract}

Received July 25, 2017.

Accepted November 20, 2017

${ }^{1}$ Corresponding author: qendrim.zebeli@vetmeduni.ac.at rumen in both concentrate phases. In conclusion, intermittent high-concentrate feeding altered the digestaassociated rumen bacterial community and rumen fermentation with more significant alterations found in Conc I than in Conc II. The data also showed that both feed additives had the most significant modulatory effects on the bacterial community, and their subsequent fermentation, during periods of low $\mathrm{pH}$.

Key words: feed additive, subacute rumen acidosis, cow-rumen bacteria, short-chain fatty acid

\section{INTRODUCTION}

The highly complex microbial community of the rumen enables multiple metabolic functions in the host. This community is evolutionarily adapted to a fiberrich herbivore diet. Yet, the feeding of large amounts of concentrates, during periods of high energy needs, may lead to rumen bacterial imbalances (McCann et al., 2016). These imbalances may further result in a decline of fiber degradation (Pourazad et al., 2016), shifts in short-chain fatty acid (SCFA) profile (Kleen et al., 2003), and accumulation of endotoxins (Plaizier et al., 2012) in the rumen, all of which can further impair cow health and productivity.

With the help of next-generation sequencing techniques, it has been possible to gain a deeper understanding of rumen bacterial shifts in cattle in response to concentrates in the diet. However, digesta-associated microbial responses to concentrate-rich diets were typically investigated in challenge studies with short and continuous feeding periods (Hook et al., 2011; Mao et al., 2013b; Petri et al., 2013), and less commonly evaluated using intermittent feeding studies. The application of an intermittent high-concentrate feeding model provides the opportunity to look at changes in the rumen microbiome and fermentation that may more closely resemble variable DMI patterns and dietary energy intake, and subsequent drops in ruminal $\mathrm{pH}$, such as during transition and peak lactation. In a recent study, we observed that cows experiencing 2 intermittent 
concentrate-rich challenges had a more severe drop in ruminal $\mathrm{pH}$ and rumination behavior during the first than during the second challenge (Kröger et al., 2017), despite feeding the same diets. These findings suggest a differing response of the ruminal microbiome during the 2 concentrate phases. More interestingly, the same experiment revealed enhancement effects on rumination behavior and ruminal $\mathrm{pH}$ in cows supplemented with phytogenic compounds (PHY) or autolyzed yeast (AY), with effects evolving especially during the first challenge phase. In vitro studies have reported PHY to have a direct bacteriostatic effect and therefore alter fermentation end products (Cardozo et al., 2005; Calsamiglia et al., 2007), whereas AY has an indirect effect by either acting as ligand for gram-negative bacteria (GNB; Nocek et al., 2011; Ganner and Schatzmayr, 2012) or as a substrate for cellulolytic bacteria (Harrison et al., 1988; Mao et al., 2013a) and so increases SCFA load (Kesselring et al., 2017). However, if the effects of intermittent concentrate feeding are related to alterations of ruminal microbiome, and whether PHY and AY supplementation modulate rumen microbiome and fermentation in an in vivo model, have not yet been evaluated.

Therefore, one aim of this study was to determine the changes of the rumen bacterial community and fermentation products, such as SCFA, ammonia, and lactate, when feeding an intermittent concentrate-rich diet to cattle. Another aim was to determine the effect of supplementation of PHY or AY feed additives on rumen bacteria shifts and the fermentation products during that intermittent high-concentrate feeding. Our hypothesis was that the increase of concentrate in the diet will decrease bacterial diversity and mediate rumen bacteria community composition and the end products of its metabolism in the rumen. Further, we hypothesized that PHY and AY will modulate the bacterial community structure in the rumen, likely resulting in a reduction in starch-fermenting bacteria and an increase in cellulolytic bacteria, leading to favorable changes in the fermentation products, such as SCFA profile. Effects are expected to be more pronounced during the first feeding phase because the time cows spent under $\mathrm{pH}$ 6.0 was longer during this phase (Kröger et al., 2017).

\section{MATERIALS AND METHODS}

\section{Animals, Diets, and Experimental Setup}

All procedures involving animal handling and treatment were approved by the institutional ethics com- mittee of the University of Veterinary Medicine (Vetmeduni) Vienna and the national authority according to $\S 26$ of the Law for Animal Experiments, Tierversuchsgesetz 2012- TVG (GZ: BMWFW-68.205/0023$\mathrm{WF} / \mathrm{V} / 3 \mathrm{~b} / 2015)$.

Detailed information about cows, feeding, and experimental setup is given in our companion paper (Kröger et al., 2017). In brief, the experiment used 8 nonlactating rumen-fistulated Holstein-Frisian cows $(863 \pm 65 \mathrm{~kg}$ of $\mathrm{BW} \pm \mathrm{SD}$ ) in an incomplete $3 \times 4$ Latin square design, balanced for carry-over effects. Cows were randomly assigned to 1 of the 3 feeding groups: control $(\mathbf{C O N}$, no feed additive), PHY (Digestarom; phytogenic product, containing herbs, spices, essential oils, and nonvolatile extracts, with major active compounds derived from mint, thyme, rosemary, and clove; BIOMIN Holding $\mathrm{GmbH}$, Getzersdorf, Austria; $3 \mathrm{~g}$ per cow per day), or AY (Levabon, Rumen E; containing yeast cell components from autolysis, as mannan oligosaccharides, glucans, chitin, nucleotides, peptides, and AA; BIOMIN Holding GmbH; $15 \mathrm{~g}$ per cow per day). Each of the 4 periods included 1 wk of baseline feeding (100\% roughage diet, RD), $6 \mathrm{~d}$ of gradual concentrate adaptation, 1 wk of high-concentrate challenge $(65 \%$ concentrates, on a DM basis, Conc I), 1 wk of recovery with roughage only, followed by a second $65 \%$ concentrate challenge lasting $2 \mathrm{wk}$ (Conc II). This feeding model was used to reflect the dietary challenge milking cows undergo during the transition period, and by using dry, nonpregnant cows, excluding any lactation-cycle-dependent influences. Feed additives were provided in the concentrate mix except during the recovery phase, where the daily doses of PHY and AY were given via the rumen cannula. Each experimental period was followed by a 3 -wk washout phase to avoid carry-over effects.

Exact protocols of feeding and feed sampling, as well as chemical composition of dietary ingredients, were described in the companion paper (Kröger et al., 2017). In brief, the RD consisted of grass silage and hay in 50:50 ratio (DM basis) and the concentrate mixture contained barley grain $(33 \%)$, wheat $(30 \%)$, rapeseed meal $(16 \%)$, corn $(15 \%)$, beet pulp $(3.2 \%)$, a mineralvitamin premix (1\%; containing $13.5 \% \mathrm{Ca}, 9 \% \mathrm{Mg}, 5 \%$ $\mathrm{P}, 1.5 \% \mathrm{Na}, 2,100,000 \mathrm{IU}$ of vitamin $\mathrm{A} / \mathrm{kg}, 300,000 \mathrm{IU}$ of vitamin $\mathrm{D} / \mathrm{kg}$, and $7,500 \mathrm{mg}$ of vitamin $\mathrm{E} / \mathrm{kg}$ ), beet molasses (1\%), calcium carbonate $(0.5 \%)$, and $\mathrm{NaCl}$ $(0.3 \%)$ on a DM basis. Diets were offered separately via individual feeding troughs equipped with computercontrolled electronic scales. Access was regulated electronically with transponder access gates (Insentec B.V., Marknesse, the Netherlands) and daily feed intake was recorded for each individual cow. 


\section{Sampling Procedures}

Rumen Microbiota. Sampling of solid digestaassociated microbiota was performed on d 6 (RD), 19 (Conc I), and 39 (Conc II) for the 3 feeding groups (n $=8) 8 \mathrm{~h}$ after morning feeding $(1600 \mathrm{~h})$. Approximately $200 \mathrm{~g}$ of solid digesta from the middle (in transversal and sagittal median of the rumen, 15 to $20 \mathrm{~cm}$ below the surface) of the rumen mat was collected via the rumen cannula, and put into a sterile plastic tray. To avoid contaminations, subsamples were taken with sterile tweezers from the middle of the sample, placed into two 2-mL Cryotubes (Sarstedt AG, Wiener Neudorf, Austria), shock frozen in liquid nitrogen, and stored at $-80^{\circ} \mathrm{C}$ until further analysis.

Rumen Fermentation Parameters. For each experimental period, sampling was performed on $\mathrm{d}$ 6 (RD), 19 (Conc I), and 39 (Conc II) for all feeding groups $(\mathrm{n}=8)$ before the morning feeding $(0600$ $\mathrm{h}), 4 \mathrm{~h}$ after the morning feeding $(1200 \mathrm{~h})$, and $8 \mathrm{~h}$ after morning feeding $(1600 \mathrm{~h})$. Approximately $500 \mathrm{~g}$ of digesta from the middle of the rumen mat was collected via the rumen cannula and manually squeezed to obtain about $250 \mathrm{~mL}$ of the particle-associated rumen liquid (PARL; Zebeli et al., 2008). The PARL samples were collected in a plastic container and the $\mathrm{pH}$ was immediately measured with a portable $\mathrm{pH}$-meter (SG2SevenGo, Mettler Toledo, Vienna, Austria). After pH measurement, the PARL sample was filtered through 4 layers of cheesecloth. From the filtered liquid, samples for SCFA, lactate, and ammonia were taken and stored at $-20^{\circ} \mathrm{C}$ until analysis.

\section{DNA Extraction and Sequencing}

Solid digesta samples were handled according to a modified protocol by (Kong et al., 2010). A 250-mg digesta sample was combined with $750 \mu \mathrm{L}$ of $0.4 \mathrm{M}$ potassium phosphate buffer in lysis A tubes (Sigma-Aldrich, Vienna, Austria) and homogenized by beat beating for $90 \mathrm{~s}(3 \times 30 \mathrm{~s})$ at speed 4.5 (Fastprep FP120, Savant Instruments Inc., Holbrook, NY). Samples were then centrifuged $10 \mathrm{~min}$ at $9,391 \times \mathrm{g}$ at room temperature and the supernatant discarded. Pre-lysis buffer $(20 \mathrm{mM}$ Tris/Cl, $2 \mathrm{~m} M$ EDTA, 1\% Triton-X 100; $\mathrm{pH} 8.0$ ) was added $(500 \mu \mathrm{L})$ to the pellet and vortexed until the pellet was resuspended. The sample was then heated at $95^{\circ} \mathrm{C}$ for $5 \mathrm{~min}$ (thermomixer compact, Eppendorf Austria GmbH, Vienna, Austria) with agitation (900 motions per minute). The tubes were centrifuged again at $18,407 \times g$ for $5 \mathrm{~min}$ at room temperature and the supernatant separated afterward in clean $2-\mathrm{mL}$ tubes, and stored on ice until DNA extraction. An amount of $1.2 \mathrm{~mL}$ of $0.4 \mathrm{M}$ potassium phosphate, $100 \mu \mathrm{L}$ of
$100 \mathrm{mg} / \mathrm{mL}$ of lysozyme, and $10 \mu \mathrm{L}$ of $2.5 \mathrm{U} / \mu \mathrm{L}$ of mutanolysin (Sigma-Aldrich) were added to the remaining pellet. The samples were then incubated at $37^{\circ} \mathrm{C}$ for 30 min (Friocell, MMM group, Munich, Germany) while shaking at 125 motions per minute. After incubation, $20 \mu \mathrm{L}$ of $18.6 \mathrm{mg} / \mathrm{mL}$ proteinase K (Sigma Aldrich, Vienna, Austria) was added and then samples were further incubated for $1 \mathrm{~h}$ at $37^{\circ} \mathrm{C}, 125$ motions per minute. Then the samples underwent a second bead-beating step $(3 \times 45 \mathrm{~s}$, speed: 4.5$)$, and were centrifuged at $14,000 \times g$ for $3 \mathrm{~min}$ at room temperature, and the supernatant was pooled with the previously separated supernatant.

Bacterial DNA was isolated using PowerSoil DNA Isolation Kit (Mobio Laboratories, Qiagen, Carlsbad, CA) according to the manufacturer's instructions with the following modifications: $250 \mu \mathrm{L}$ of the prepared sample was used; after adding solution $\mathrm{C} 1$, the tubes were vortexed for only $1 \mathrm{~min}$ (instead of $10 \mathrm{~min}$ ) on the vortex adapter (vortex genie2, Scientific Industries Inc., Bohemia, NY); to elute DNA, $2 \times 50 \mu \mathrm{L}$ of $70^{\circ} \mathrm{C}$ sterile DNA-Free PCR grade Water (DEPC treated water, Thermo Fisher Scientific Inc., Waltham, MA) was used. After DNA extraction, samples were stored at $-20^{\circ} \mathrm{C}$. Total DNA quantity after isolation was measured for all samples using a Qubit Fluorometer 2.0 (Qubit dsDNA HS Assay Kit, Thermo Fisher Scientific, Vienna, Austria) according to the manufacturer's instructions. Amplicon sequencing was performed using Illumina MiSeq paired-ends sequencing technology (Microsynth AG, Balgach, Switzerland). The hypervariable region V3 to V5 of bacterial 16S rRNA genes was amplified by bridge amplification using the primer set 357 forward (5'-CCTACGGGAGGCAGCAG-3'), and 926 reverse (5'-CCGTCAATTCMTTTRAGT-3'; Peterson et al., 2009) to generate an approximate amplicon size of 570 bp. Multiplexed libraries were constructed by ligating sequencing adapters and indexes onto purified PCR products using the Nextera XT Sample Preparation Kit (Illumina). Primers were trimmed and corresponding overlapping paired-end reads were stitched by Microsynth (Microsynth AG).

\section{Sequence Processing}

The sequencing data were deposited into the European Nucleotid Archive and can be accessed via accession number PRJEB21562 (http://www.ebi.ac.uk/ ena/data/view/PRJEB21562). Sequence data were analyzed with the open source bioinformatics pipeline QIIME (version 1.9.1. http://qiime.org/), based on the recommended workflow of QIIME tutorials (http:// qiime.org/tutorials/index.html; last accessed November 14, 2016) and Navas-Molina et al. (2013). Briefly, 
sequences of low quality were trimmed with a quality score of 20 . The chimeric sequences were identified using the gold.fa reference database (Mukherjee et al., 2017), and subsequently filtered. A total of 1,182,865 sequences passed the quality control and chimera check. Sequences were then clustered into operational taxonomic units (OTU) with a $97 \%$ 16S rRNA gene similarity cutoff by performing open reference OTU picking. The database of SILVA (https://www.arb -silva.de, version 123; last accessed August 25, 2016) was used for taxonomic classification. The OTU with less than 10 sequences in total were excluded for data normalization. A total of 5,400 OTU were found; however, 47 OTU were unassigned at kingdom level and were therefore removed. The remaining 5,353 OTU, with a total number of 1,181,185 sequences, were used for taxonomic-based analyses. The percent relative abundances of all OTU were calculated and OTU were ranked according to their abundance. Secondary taxonomic classification was performed for highly abundant OTU using the Basic Local Alignment Search Tool (BLAST) of the National Center for Biotechnology Information database (https://blast.ncbi.nlm.nih.gov/ Blast.cgi; last accessed April 1, 2017). Measures of $\alpha$ diversity were also determined using QIIME, specifically nonparametric species richness estimator Chao 1, as well as the diversity indices Shannon and Simpson. The rarefaction depth was equalized for all samples to 6,245 sequences per sample based on the minimum sequence number achieved. For $\beta$-diversity analysis, the weighted UniFrac distance matrix (Lozupone and Knight, 2005) was also calculated with the same normalization of sequences per sample. Venn diagrams were created using Venn Diagram Plotter (version 1.5., PNNL, Richland, WA) and Venny (version 2.1., Oliveros, 2007). Sequencing results were analyzed at the genus level to overview the changes of the whole population. For interpreting changes of the highly dominant taxa, the most abundant 100 OTU with their taxonomic assignment of $\geq 97 \%$ sequence similarity to SILVA or NCBI were chosen. The assignment to gram-negative and gram-positive bacterial cell wall was based on literature (Dworkin, 2006a,b; Whitman, 2009).

\section{Rumen Fermentation Variables}

Concentrations of SCFA (acetate, propionate, butyrate, iso-butyrate, valerate, iso-valerate, caproate) were determined by GC as previously described by Qumar et al. (2016). Briefly, the samples were centrifuged at $20,000 \times g$ for $25 \mathrm{~min}$ at $4^{\circ} \mathrm{C}, 0.2 \mathrm{~mL}$ of $\mathrm{HCl}(1.8 \mathrm{~mol} / \mathrm{L})$ was added to the supernatant, followed by addition of $0.2 \mathrm{~mL}$ of the internal standard (4-methylvaleric acid, Sigma-Aldrich Co. LLC, St. Louis, MO). The mixture was centrifuged at $20,000 \times g$ for $25 \mathrm{~min}$ at $4^{\circ} \mathrm{C}$ to remove any precipitated substrates. The clear supernatant was transferred into the GC vial and was analyzed for SCFA concentrations via GC apparatus (GC Model 8060 MS172DPFC, no. 950713, Fisons, Rodena, Italy). Injector and detector were at temperatures of 170 and $190^{\circ} \mathrm{C}$, respectively, and helium was used as a carrier gas with a flowrate of $6 \mathrm{~mL} / \mathrm{min}$. As reference, an external standard for each targeted acid with a known concentration was used. Generation and evaluation of chromatograms was done with Stratos Software (Stratos Version 4.5.0.0, Polymer Laboratories, Church Stretton, Shropshire, UK).

The ammonia concentration in the PARL samples was determined using the indophenol reaction (Weatherburn, 1967). For this analysis, the samples were thawed at room temperature and then centrifuged at $15,115 \times$ $g$ for $10 \mathrm{~min}$ at room temperature. Subsequently, the clear supernatant was diluted with distilled water to obtain a concentration within the standard calibration curve. Both ammonia and phenol were oxidized by sodium hydroxide in the presence of sodium nitroprusside and dichloroisocyanuric acid to form a blue complex. After 90 min of reaction, the absorbance was measured at $655 \mathrm{~nm}$ using U3000 Spectrophotometer (INULA GmbH, Vienna, Austria). The blue color intensity was linearly correlated with ammonia concentration of the sample.

Lactic acid contents of the PARL samples were analyzed photometrically using an enzymatic test kits according to the manufacturer's instructions (Megazyme D-, L-lactate assay kit; Megazyme International, Wicklow, Irland) for $8 \mathrm{~h}$ after morning feeding only.

\section{Statistical Analyses}

Statistical analyses for SCFA, ammonia and lactate content, and genus and OTU taxonomy were performed using SAS (version 9.2, SAS Institute Inc., Cary, NC). Overall, the treatments and blocking variables did not interact, as assumed for the Latin square design. Data were checked for normality and variance homogeneity before further statistical analysis. For variables that did not meet the assumptions required for ANOVA, generalized linear mixed models were applied (GLIMMIX procedure of SAS), whereas the rest of the data were analyzed using the MIXED procedure of SAS (version 9.2, SAS Institute Inc.). All statistical models were performed with the feeding phase (RD, Conc I, and Conc II) and feeding group (CON, PHY, AY) considered as fixed effects. Interactions between feeding phase and feeding group (phase $\times$ group) were also analyzed. For variables of SCFA and ammonia, the fixed effect of sampling hour after the morning feeding 
as well as the interaction between sampling hour and feeding phase (hour $\times$ phase) was also accounted in the model. To account for repeated measurements of the same animal within a feeding group over time, a variance-components variance-covariance structure was used according to the Bayesian information criterion. Cow nested within square and the experimental period was considered as a random effect. Treatment effects are only given for feeding phases Conc I and Conc II because feed additives were only administered during those phases. Comparisons among the least squares means were performed with pdiff option and considered as significant with $P<0.05$ and as a trend with $0.05 \leq P \leq 0.10$. Principal coordinate analysis of the Weighted Unifrac distance matrix data was performed using PROC PRINCOMP of SAS (version 9.2, SAS Institute Inc.). Correlations between the fermentation parameters and the microbiota data of the most abundant 100 OTU were analyzed using Pearson correlation coefficient (PROC CORR of SAS). According to Hinkle et al. (2003), the $\mathrm{r}$ was interpreted as follows: 0.00 to 0.30 as negligible; 0.30 to 0.50 as low; 0.50 to 0.70 as moderate; 0.70 to 0.90 as high, and 0.90 to 1.00 as substantial. For our interpretation we considered moderate, high, or substantial correlations from $\mathrm{r}>0.5$.

\section{RESULTS}

The influences of concentrate feeding and the feed additives on DMI, minimum reticuloruminal $\mathrm{pH}$, and duration of $\mathrm{pH}<6.0$ have been previously published in the companion study (Kröger et al., 2017), and are used in this article only for correlating with the current microbiological data. The objectives of the current study were to evaluate how an intermittent high-concentrate feeding model and the supplementation of PHY or AY feed additives alters the rumen bacterial community and rumen fermentation parameters such as SCFA, ammonia, and lactate.

\section{Effect on Bacterial Population Diversity and Phylogeny}

Data of $\alpha$-diversity indices are shown in Figure 1. The Shannon index was decreased with concentrate feeding compared with RD (Figure 1A). Both Chao 1 (Figure $1 \mathrm{~B})$ and the Simpson index (Figure 1C) were even further decreased in Conc I compared with Conc II $(P<$ 0.01). All 3 parameters had a lower standard deviation for RD than for Conc I and Conc II. In the PHY group (0.908), Simpson index showed a significant $(P=0.02)$ decrease compared with CON (0.947) during Conc I. Principal coordinate analysis of the Weighted Unifrac distance matrix revealed a clear clustering of the $\mathrm{RD}$ samples. Moreover, the RD cluster was located apart from the 2 concentrate groups with a high variation of $52 \%$ along the principal component 1 axis (Figures $2 \mathrm{~A}$ and $2 \mathrm{C}$ ). The samples of the 2 concentrate phases showed a more widespread clustering within their samples (Figures 2A, 2B, and 2C). UniFrac distance matrix analysis showed no clustering according to the 3 feeding groups CON, PHY, and AY. Between the 3 feeding phases, 2,646 of the total 5,353 OTU were shared (Figure 3). The RD had the highest number of unique OTU and the 2 concentrate phases shared the highest number of OTU.

According to the OTU assignment a total of $35.08 \%$ of the OTU could be assigned to 53 genera, 46 of them being affected by the concentrate feeding. The 2 concentrate challenges Conc I and Conc II decreased most

\section{A Shannon evenness estimation}

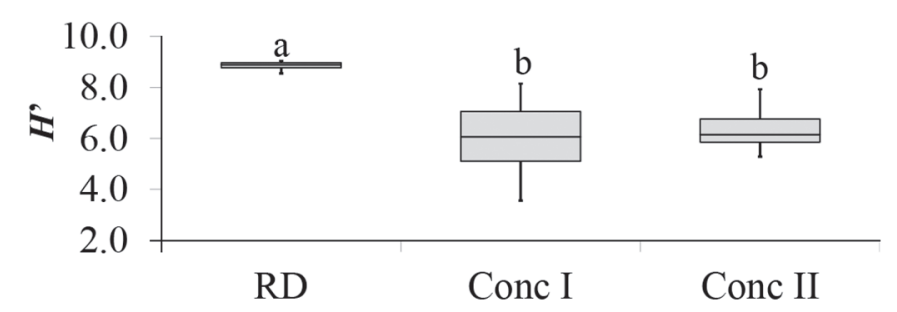

B
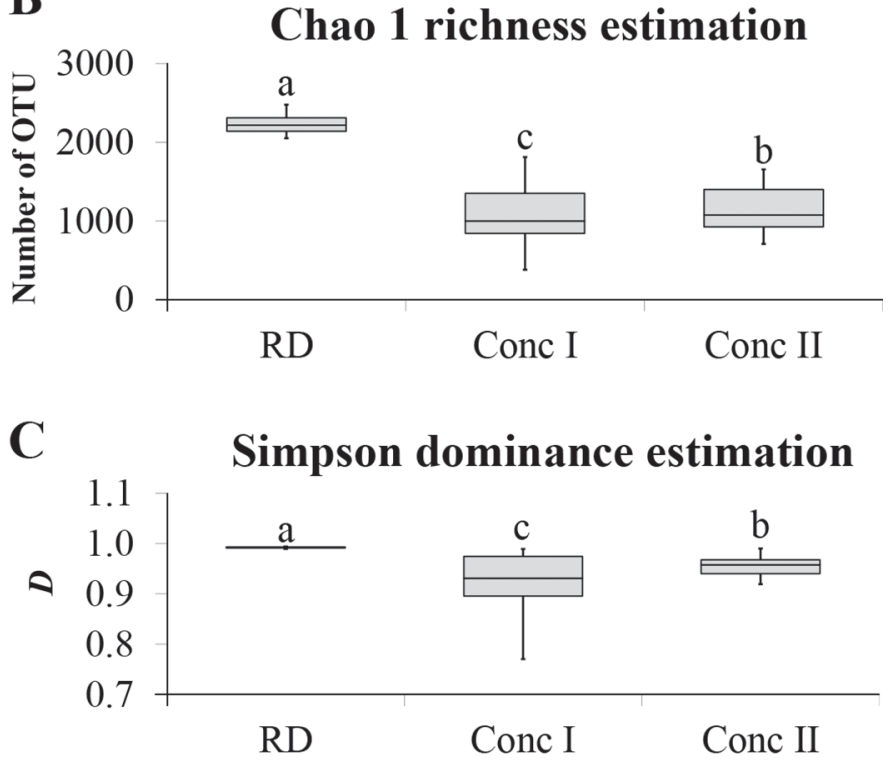

Feeding phase

Figure 1. Alpha diversity indices Shannon (A), Chao 1 (B), and Simpson (C) derived from the QIIME (http://qiime.org/) command $\alpha$ rarefraction. Different lowercase letters a, b, and c indicate significant $(P<0.01)$ changes between feeding phases roughage diet for $1 \mathrm{wk}$ (RD), $65 \%$ concentrates for $1 \mathrm{wk}$ (Conc I), and $65 \%$ concentrates for $2 \mathrm{wk}$ (Conc II). OTU = operational taxonomic unit; upper box $=2 \mathrm{nd}$ quartile; mid line $=$ median; lower box $=3 \mathrm{rd}$ quartile; whiskers $=$ highest and lowest quartile. 

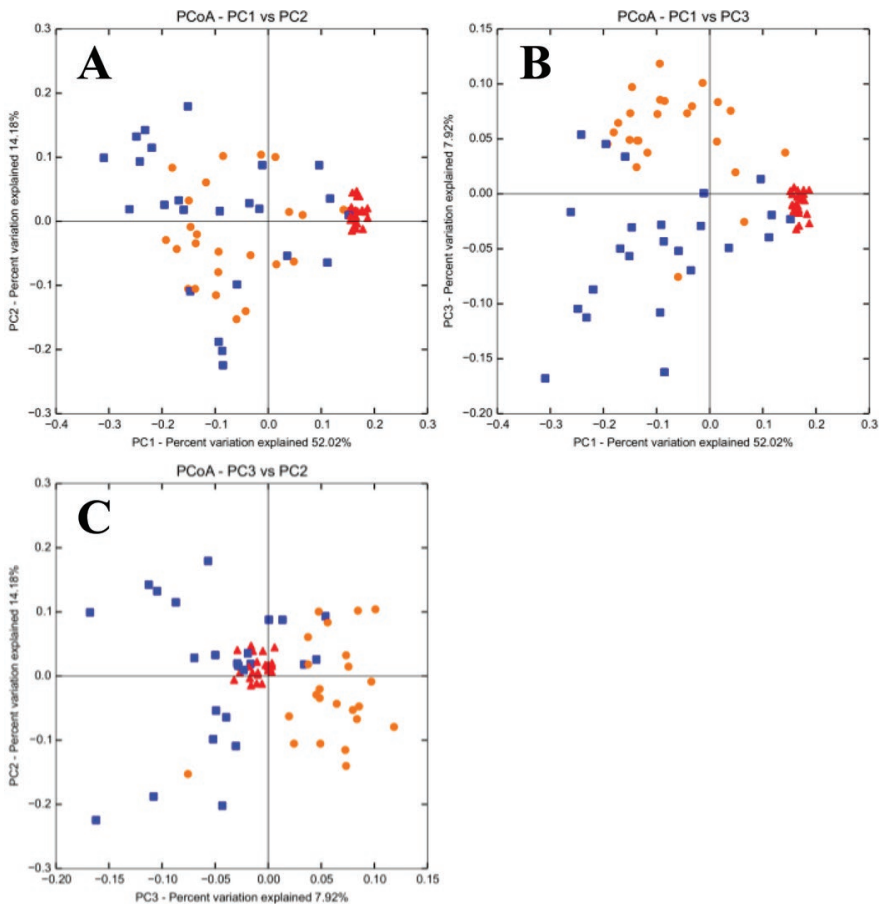

Figure 2. (A, B, C) Two-dimensional principal coordinate analysis (PCoA) plots based on weighted UniFrac distance matrices, showing the phylogenetic clustering according to the 3 feeding phases $1 \mathrm{wk}$ roughage diet (RD; triangle), 1 wk $65 \%$ concentrates (Conc I, square), and $2 \mathrm{wk} 65 \%$ concentrates (Conc II; circle). The 3 axes [principal component (PC) 1, 2, 3] indicate the variation between the samples in percent. Color version available online.

of the genera (39 and 41 genera, respectively) and increased only 14 and 12 genera, respectively, in comparison to RD (Supplemental Figure S1; https://doi.org/10 $.3168 /$ jds.2017-13565). When looking at the differences in relative abundance between the 2 concentrate feeding phases, 8 genera were higher in Conc II than Conc I and 7 genera lower in Conc II than in Conc I. Changes in percent abundance, both positive and negative, were identical for both high-concentrate challenges relative to RD for all genera. The most abundant genus, Prevotella ( $12.7 \%$ total relative abundance), was increased by $49 \%$ $(P<0.01)$ in both concentrate challenges, as well as Shuttleworthia, which increased by $98 \%$ in Conc I $(P<$ $0.01 ; 0.9 \%$ total relative abundance). Most of the other high abundance genera such as Ruminococcus $(5.4 \%$ total relative abundance), Butyrivibrio (4.6\%), Coprococcus (2.2\%), Blautia (1.0\%), and Pseudobutyrivibrio $(0.8 \%)$ decreased $(P \leq 0.03)$ with concentrate feeding by $49,37,24,88$, and $79 \%$, respectively. Within the less abundant genera, Fibrobacter $(0.2 \%)$ decreased by $70 \%$ $(P<0.01)$ in the 2 concentrate diets, whereas others, such as Lachnospira (0.2\%), Megasphaera (0.2\%), Lactobacillus (0.08\%), Succinivibrio $(0.06 \%)$, and Bacteroides $(0.04 \%)$ significantly increased $(P \leq 0.03)$ at least in 1 of the 2 concentrate phases by $63,100,100,88$, and
100\%, respectively (Supplemental Figure S1; https:// doi.org/10.3168/jds.2017-13565). Both feed additives PHY and AY had effects on both high and low abundance genera, most of them occurring during Conc I (Figure 4A and 4B). Autolyzed yeast significantly increased Ruminococcus $(P<0.01)$ and Mogibacterium $(P<0.05)$, tended to increase Clostridium $(P=0.08)$ and Dorea $(P=0.06)$, and tended to decrease Methylobacterium $(P=0.07)$. Both PHY and AY decreased low abundance genera such as Treponema $(P<0.01)$ and Bacteroides $(P<0.01$ for PHY, $P=0.07$ for AY $)$, and decreased Anaerovorax $(P=0.07)$, and Fusobacterium $(P<0.08)$ with a trend. Moreover, PHY had a significant decreasing effect on Roseburia $(P=0.03)$, YRC22 $(P=0.02)$, and a trend to decrease Bifidobacterium $(P<0.07)$, and a significant increasing effect on Oscillospira $(P=0.02)$ and Curtobacterium $(P=0.02)$ and an increasing trend on Anaerostipes $(P=0.05$; Figures $4 \mathrm{~A}$ and $4 \mathrm{~B})$.

Supplemental Figure S2 (https://doi.org/10.3168/jds .2017-13565) provides a list with the first most abundant 100 OTU, representing those with a total relative abundance of $\geq 0.15 \%$, and their changes of abundance along the 3 feeding phases RD, Conc I, and Conc II. Overall, only 9 out of the 100 most abundant OTU were not affected by the concentrate feeding. Statistically significant changes in relative abundance between the 2 Conc phases occurred in 44 OTU. Similar to the genera data, Conc I and Conc II always showed the same abundance changes in comparison to $\mathrm{RD}$, apart

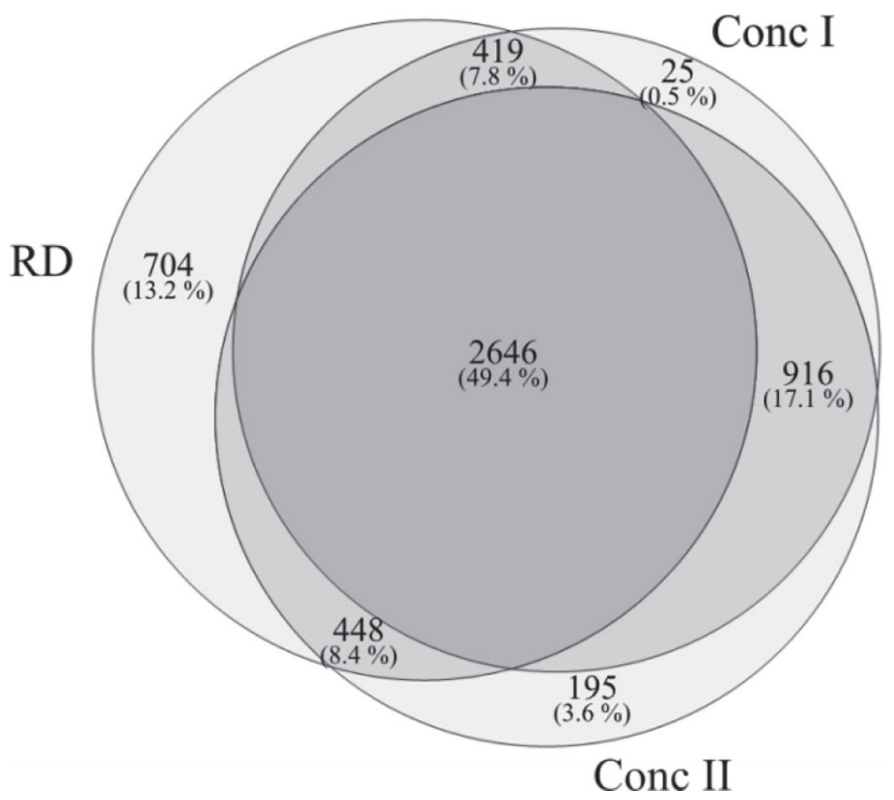

Figure 3. Venn diagram illustrating the number and percentage of individual and shared operational taxonomic units (OTU) of the 3 feeding phases: $100 \%$ roughage diet for $1 \mathrm{wk}(\mathrm{RD})$, and $65 \%$ concentrates for $1 \mathrm{wk}$ (Conc I) and $2 \mathrm{wk}$ (Conc II). 


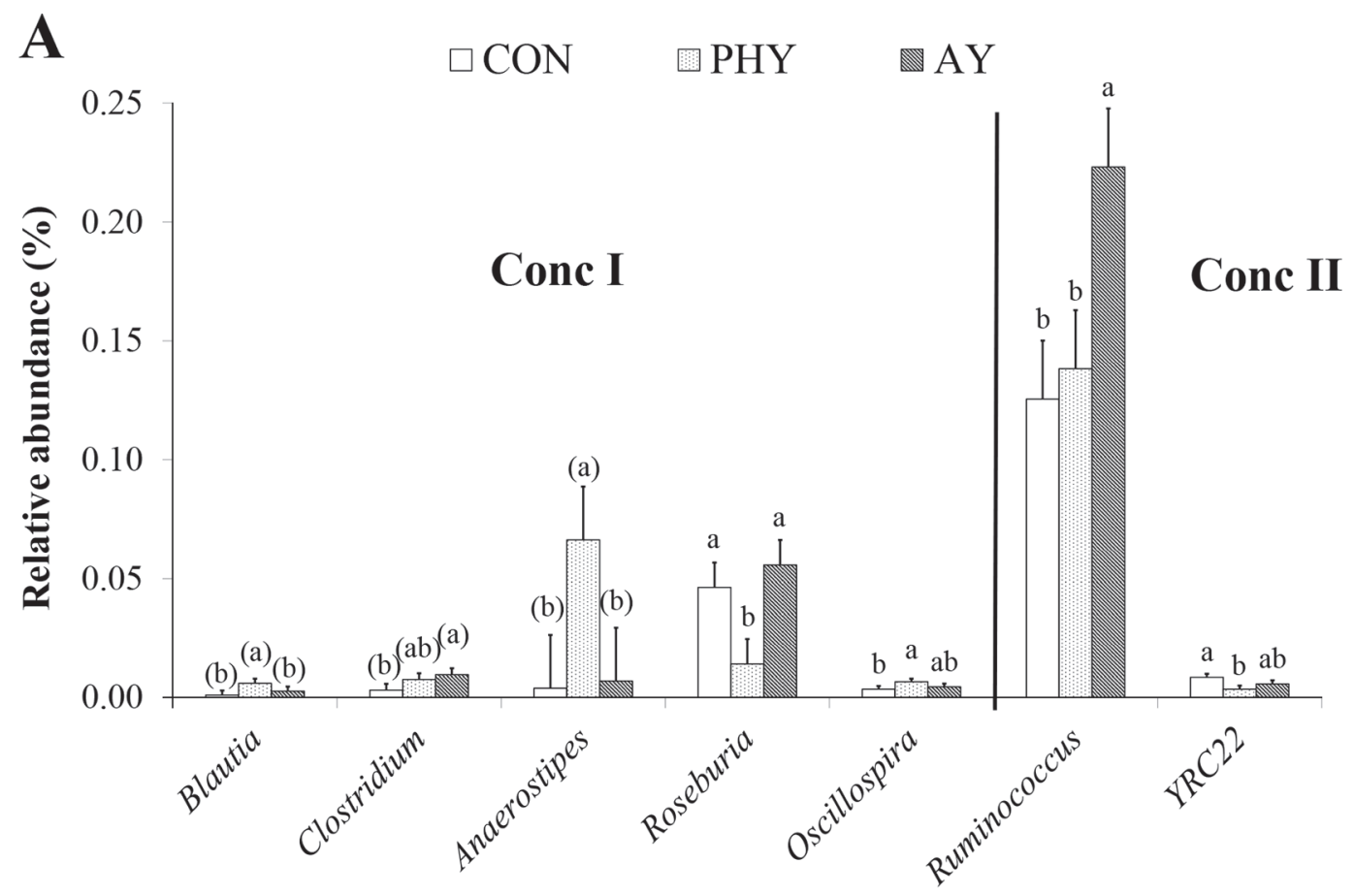

High abundance genera

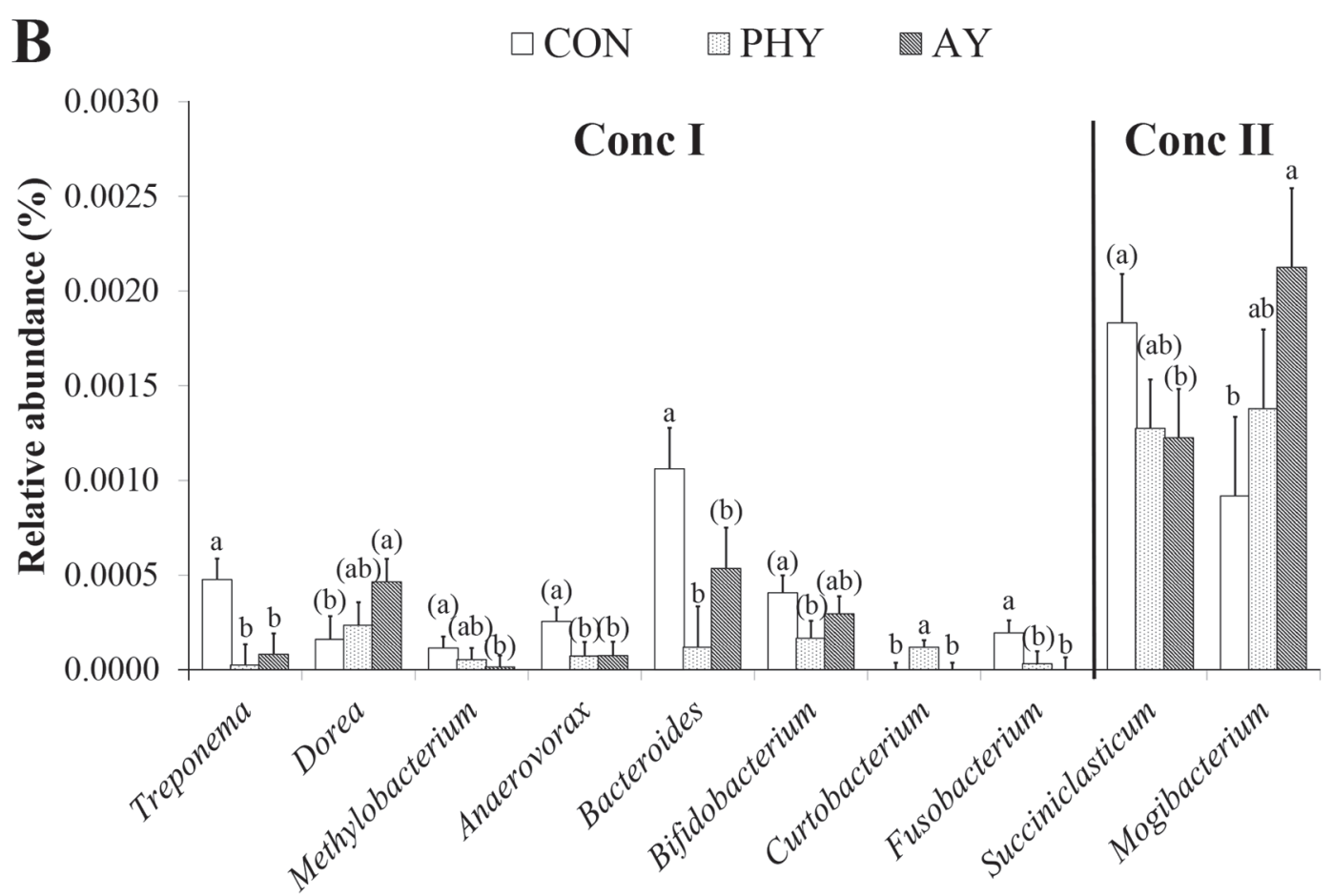

\section{Low abundance genera}

Figure 4. (A, B) Effect of the additives phytogenic (PHY) and autolyzed yeast (AY) on high (A) and low (B) abundance genera during 65\% concentrate for 1 and $2 \mathrm{wk}$ (Conc I and Conc II, respectively). CON = control. Different lowercase letters indicate significant differences between the feed additives $(P<0.01)$, and lowercase letters in parentheses indicate a trend $(0.05<P<0.1)$. Error bars indicate SEM. 
from 1 OTU; the Butyrivibrio fibrisolvens OTU_62 was significantly higher in Conc I $(P<0.01)$ and had a trend to be lower in Conc II $(P=0.08)$ in comparison to RD. From the most taxonomically similar OTU found, 20 Prevotella spp. associated OTU increased with concentrate feeding, 2 did not differ between the feeding phases and only one single Prevotella OTU decreased in the 2 concentrate phases. The Clostridiales order (97\% similarity) was represented by 7 OTU, including the most abundant OTU_1, and was increased in 5 instances from RD to concentrate feeding. Ruminococcaceae (10 OTU) and Ruminococcus spp. (12 OTU) were decreased in 16 cases and increased in 4 OTU. Lachnospiraceae family was represented by 12 OTU, most of them increasing with concentrate feeding. Butyrivibrio spp. decreased in 5 out of 8 OTU with concentrate feeding. Within the 100 most abundant OTU, PHY and AY showed effects only on the OTU in Conc I as compared with CON, except an increasing effect of AY on Ruminococcus flavefaciens (OTU_23) in Conc II (Figure 5). The 4 Ruminococcaceae family OTU were increased by PHY, whereas Prevotella spp. associated OTU were decreased by PHY and AY. Additionally, one Ruminococcus spp. (OTU_48) was increased by AY in Conc I (Figure 5).

To elucidate shifts of major bacterial groups of the rumen, we analyzed the data according to known Gram staining of the genera found using taxonomic classification and found $50 \%$ to be gram-positive bacteria (GPB) and $31 \%$ GNB. The rest were of variable or undefined Gram staining (Supplemental Figures S1 and S2; https://doi.org/10.3168/jds.2017-13565).

\section{Effect on Rumen Fermentation Profile}

The percentage of acetate, iso-butyrate, caproate, acetate:propionate ratio, and PARL $\mathrm{pH}$ were significantly $(P<0.05)$ decreased in both high-concentrate challenges in comparison with the RD (Figure 6; Supplemental Table S1; https://doi.org/10.3168/jds.2017 -13565). Propionate, butyrate, and valerate increased in both concentrate challenges in comparison to the RD level $(P<0.01)$. Propionate increased even further from Conc I to Conc II, whereas butyrate decreased slightly from Conc I to Conc II but did not return to RD levels $(P<0.01)$. The PHY group had a higher percentage of butyrate in both concentrate challenges (Conc I, $P=0.01$; Conc II, $P=0.08$ ), higher trend for iso-valerate in Conc I, and lower trend for propionate, and significantly lower valerate and caproate in Conc I compared with CON (Table 1). The AY supplementation group showed significantly lower valerate and caproate in Conc I, whereas a higher trend for valerate in Conc II, and significantly lower acetate and iso-valerate in Conc II compared with CON. Sampling time point affected propionate and butyrate differently depending on the feeding phase (Figure 6). Acetate and the acetate:propionate ratio decreased with time after morning feeding and were significantly different $8 \mathrm{~h}$ after feeding $(P<0.01)$ for RD and Conc I. No effect of feed additives on acetate:propionate ratio was seen. Total lactate, as well as the fractions of D-lactate and L-lactate, were increased in both concentrate phases $(P<0.04)$, and the fraction of L-lactate showed an increasing trend in the AY group during Conc I (Table 1). Ammonia concentration was decreased significantly in Conc II in comparison to the RD level $(P=0.03$; Supplemental Table S1; https://doi.org/10.3168/jds .2017-13565), without any effects of feed additives. Total SCFA showed no overall difference between RD and the concentrate phases $(P=0.69)$ or feed additives $(P=$ 0.47 ), but when considering the different sampling time points, total SCFA was higher in RD $4 \mathrm{~h}$ after feeding and highest in both concentrate phases $8 \mathrm{~h}$ after feeding $(P<0.01$; Figure 6$)$. Particle-associated $\mathrm{pH}$ showed no time point effect in $\mathrm{RD}$, but decreased significantly $8 \mathrm{~h}$ after feeding in both concentrate phases $(P<0.01)$.

Correlation analysis was performed for the first 100 OTU and the evaluated fermentation parameters, as well as $\mathrm{pH}$ and DMI (Figure 7). For most OTU, a positive correlation was observed with mean reticular and PARL pH, as well as acetate and iso-butyrate. Conversely, a negative correlation was observed with concentrate intake $(\mathrm{DM})$, time $\mathrm{pH}<6.0$, propionate, butyrate, and valerate. Caproate also had positive moderate correlations with Shuttleworthia spp., Syntrophococcus spp., and Olsenella spp. OTU. For total SCFA, iso-valerate, lactate, and $\mathrm{NH}_{3}$, only 1 OTU showed a moderate correlation for each parameter, being OTU_29 Lachnospiraceae spp., OTU_95 Moryella spp., OTU_73 Prevotella spp., and OTU_32 Prevotella bryantii [best Basic Local Alignment Search Tool nucleotide (BLASTn) hit, 100\% similarity], respectively (Figure 7).

\section{DISCUSSION}

\section{Effect of Feeding 2 Intermittent High-Concentrate Diets}

The main aim of this study was to determine rumen bacterial community changes and fermentative effects of feeding an intermittent concentrate-rich diet in cattle. The growth, activity, and homeostatic regulation of rumen microbes depends mainly on the substrate availability and specificity of rumen microbes (Henderson et al., 2015). We found that the change from forage-only diet to a high-grain diet clearly de- 


\begin{tabular}{|c|c|c|c|c|c|c|c|c|c|}
\hline \multirow{3}{*}{$\begin{array}{l}\text { OTU } \\
\text { Number }\end{array}$} & \multirow{3}{*}{$\begin{array}{l}\text { Taxonomic classification } \\
\text { according to SILVA and NCBI } \\
\text { best BLASTn hit } \geq 97 \% \text { identity }\end{array}$} & \multirow{3}{*}{$\begin{array}{c}\text { Identity } \\
\% \\
\end{array}$} & \multicolumn{6}{|c|}{ Feeding phase } & \\
\hline & & & \multicolumn{3}{|c|}{ Conc I } & \multicolumn{3}{|c|}{ Conc II } & \\
\hline & & & CON & PHY & AY & CON & PHY & AY & \\
\hline OTU_6 & Ruminococcaceae (f) & 12 & & $* *$ & & & & & \\
\hline OTU_14 & Syntrophococcus (g) & 100 & & $* *$ & & & & & \\
\hline OTU_17 & Ruminococcaceae (f) & 97 & & $* *$ & & & & & \\
\hline OTU_21 & Prevotella ruminicola (s) & 100 & & $* *$ & $*$ & & & & \\
\hline OTU_23 & Ruminococcus flavefaciens (s) & 99 & & & & & & * & \\
\hline OTU_26 & Saccharofermentans (g) & 100 & & & $* *$ & & & & \\
\hline OTU_31 & Ruminococcaceae (f) & 97 & & $* *$ & & & & & \\
\hline OTU_48 & Ruminococcus (g) & 97 & & & $* *$ & & & & Relative abundance $\%$ \\
\hline OTU_50 & Prevotella ruminicola (s) & 99 & & $* *$ & $* *$ & & & & $\geq 2.0$ \\
\hline OTU_52 & Roseburia intestinalis (s) & 97 & & & $*$ & & & & $1.0-2.0$ \\
\hline OTU_59 & Shuttleworthia (g) & 97 & & $* *$ & & & & & $0.5-1.0$ \\
\hline OTU_60 & Ruminococcaceae (f) & 97 & & $* *$ & & & & & $0.2-0.5$ \\
\hline OTU_76 & Prevotella (g) & 99 & & $* *$ & & & & & $<0.2$ \\
\hline OTU_95 & Moryella indoligenes (s) & 96 & & $* *$ & & & & & $* * \quad P<0.01$ \\
\hline OTU_99 & Olsenella (g) & 99 & & $* *$ & $* *$ & & & & $0.01<P<0.05$ \\
\hline OTU_100 & Eubacterium pyruvativorans (s) & 99 & & $* *$ & & & & & \\
\hline
\end{tabular}

Figure 5. Heatmap showing the influence of the feed additives phytogenic (PHY) and autolyzed yeast (AY) compared with control (CON) onto the first most abundant 100 operational taxonomic units (OTU). Only feeding-phase $\times$ feed-additive interactions that had $P<0.1$ for the feed additive contrast or fixed effects feed-additive were considered in this table. Based on the higher sequence identity to the reference database, either results from OTU picking against SILVA (https://www.arb-silva.de, version 123; last accessed August 25, 2016) or the best Basic Local Alignment Search Tool nucleotide (BLASTn) hit against the National Center for Biotechnology Information was chosen as the taxonomic reference. The letter in parentheses is indicative for the taxonomic level reached with at least $97 \%$ sequence identity: family (f), genus (g), and species $(\mathrm{s})$. Conc $\mathrm{I}=65 \%$ concentrate $(\mathrm{DM})$ for $1 \mathrm{wk}$; Conc II $=65 \%$ concentrate $(\mathrm{DM})$ for 2 wk. Color version available online.

creased rumen bacteria diversity (Mao et al., 2013b). Interestingly, the second concentrate challenge had a higher Simpson index, higher number of unique OTU, and a separated phylogenetic clustering in comparison to Conc I. This indicates that Conc I had a stronger effect, with a clearly disrupted bacterial community, than Conc II, despite the same diet was fed. During 1 wk of roughage-only break, the bacterial community seemed to more closely resemble a high-grain diet microbiome than a roughage microbiome (Petri et al., 2013), which would underline the adaptation of the rumen microbiome to the concentrate diet in the second challenge. Principal coordinate analysis showed that RD samples clearly form a separate cluster against concentrate diet samples, which suggests that the bacterial community was able to return to the initial RD composition within 3 wk of washout and 1 wk of RD (Hook et al., 2011).

The findings of distribution of GPB (50\%) and GNB $(31 \%)$ in the digesta samples corresponded with other studies (53\% GPB and 41\% GNB) using 40\% concentrate as control and $70 \%$ concentrate as challenge (Mao et al., 2013b). Mao et al. (2013b) further found that the relative abundance of GPB was higher in the $70 \%$ concentrate group than in the control group. In the current study, the GNB increased with concentrate feeding and had at genus level numerically a higher mean relative abundance $(2.45 \%)$ in the concentrate phases than GPB (1.79\%). These results are mainly driven by the increase of the most prevalent genus in our study, Prevotella, a gram-negative rod, which is known to be found in high abundance (42-60\%), and high genetic variability in the rumen (Stevenson and Weimer, 2007). Previous research has shown variable levels of Prevotella with relation to dietary change (Mao et al., 2013b). However, with the use of sequence data, it must be considered that taxonomic databases are based predominantly on cultured and fully sequenced species of Prevotella such as P. bryantii, P. ruminicola, and P. brevis, which represent only 2 to $4 \%$ of all Prevotella found in the rumen (Stevenson and Weimer, 2007). Therefore, it is highly probable that other uncultured fastidious Prevotella species have variable substrate specificities and are capable of growing under low pH conditions (Purushe et al., 2010). A similar contradiction was seen with another highly abundant GNB OTU, Syntrophococcus spp., which increased in 

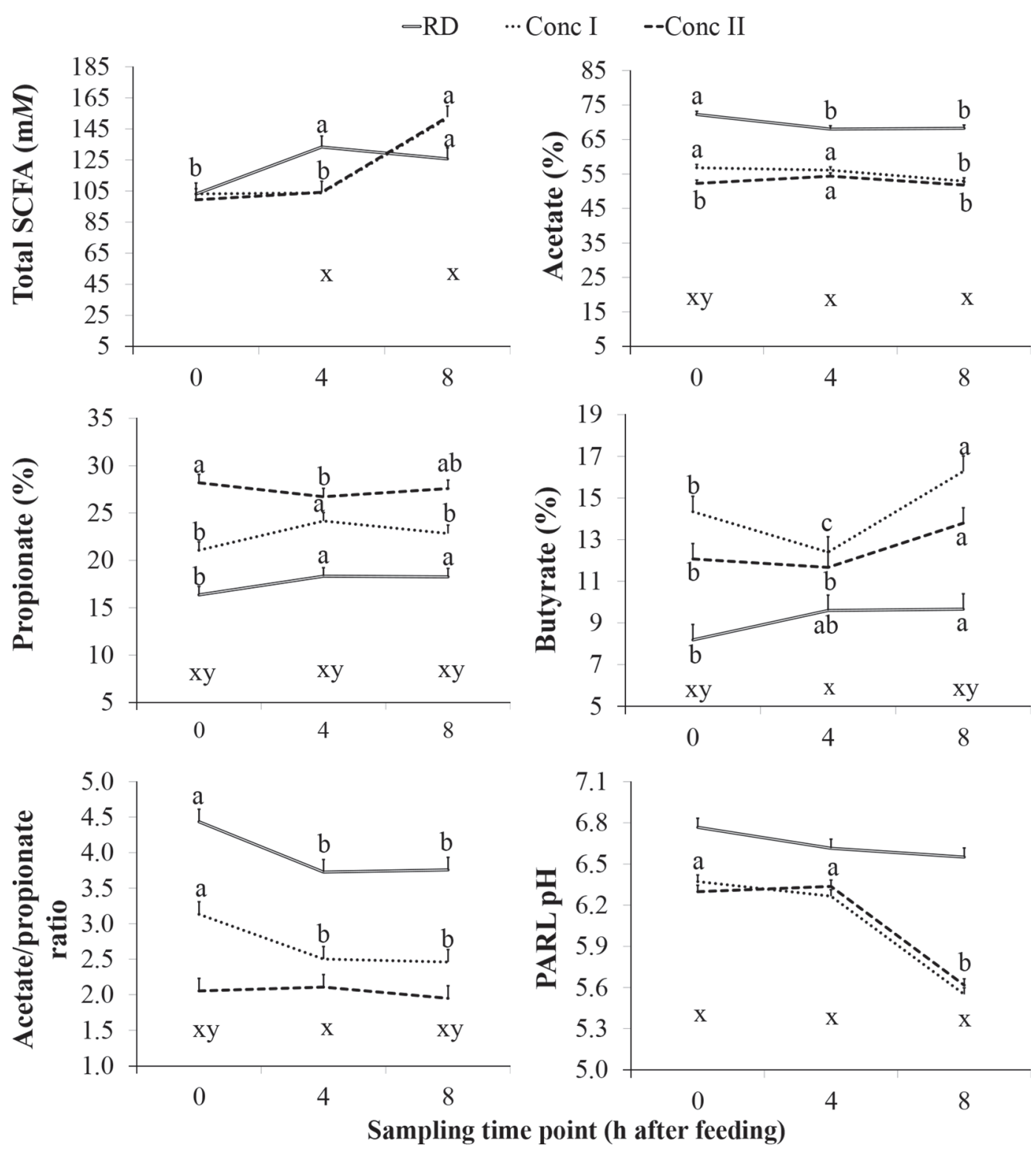

Figure 6. Effect of sampling time point on total short-chain fatty acid (SCFA) amount acetate, propionate, and butyrate proportion; acetate:propionate ratio, and particle-associated rumen liquid (PARL) pH during $100 \%$ roughage for 1 wk (RD), $65 \%$ concentrates for 1 wk (Conc I), and $65 \%$ concentrates for 2 wk (Conc II). The lowercase letters a, b, and c indicate significant $(P<0.01)$ changes between the 3 sampling time points 0 (before feeding), 4 ( $4 \mathrm{~h}$ after morning feeding), and 8 ( $8 \mathrm{~h}$ after feeding). The lowercase letters $\mathrm{x}$ and $\mathrm{y}$ indicate a significant $(P<0.01)$ difference between $\mathrm{RD}$ and the 2 Conc diets $(\mathrm{x})$ or between Conc I and Conc II $(\mathrm{y})$ during the sampling time points.

our study with concentrate feeding. Syntrophococcus is identified as a genus that preferentially grows in a $\mathrm{pH}$ range of 6.0 to 7.6 (Whitman, 2009). Because the $\mathrm{pH}$ in our study was found to be below 6.0 for extended duration of time under high-concentrate feeding (Kröger et al., 2017), these results suggest that the $\mathrm{pH}$ sensitivity of certain GNB and GPB species needs to be reevaluated (Nagaraja and Titgemeyer, 2007). This research question is of particular interest due to the release of possible toxic LPS from GNB, as it is known for the
GNB Escherichia coli, causing both local and systemic inflammation (Khafipour et al., 2009).

The shift from acetate toward more propionate production and lower $\mathrm{pH}$ conditions in the rumen underlies the changing nutrient supply and subsequent metabolism of rumen bacteria during the transition from a forage to a high-concentrate diet (Kleen et al., 2003; Dijkstra et al., 2012). According to our findings, Ruminococcus spp., as well as other acetate producing genera including Saccharofermentans, Blautia, and 
Dorea (Liu et al., 2008; Whitman, 2009; Chen et al., $2010)$, correlated $(r>0.5)$ with rumen acetate levels in the $\mathrm{RD}$, and correspondingly decreased with concentrate feeding. Additionally, the increased abundance of known propionate producers, including Prevotella spp., Bacteroides spp., Olsenella spp., Succinivibrio spp., and Megasphaera spp. (Dewhirst et al., 2001; Whitman, 2009; Purushe et al., 2010), correlated with the increased propionate levels during concentrate feeding. Furthermore, the acetate:propionate ratio continued to decrease from Conc I to Conc II, which is supported by similar changes in the microbial population, either significantly or numerically, despite rumen $\mathrm{pH}$ being higher in Conc II than in Conc I. The continued decrease in acetate:propionate ratio without a decrease in $\mathrm{pH}$ indicates that $\mathrm{pH}$ was able to recover to a certain point in Conc II due to higher salivary buffers (Kröger et al., 2017), or higher epithelial absorption and buffering (Aschenbach et al., 2011), whereas microbial groups and their metabolism were even more in favor of propionate production in Conc II. These results suggest that dietary substrates and ruminal $\mathrm{pH}$ are not solely responsible for metabolic alterations of the microbiome (van Houtert, 1993; Dijkstra et al., 2012), but other influencing mechanisms took place during Conc II feeding.

The butyrate percentage was increased with concentrate feeding, with the greatest increase during Conc I and an intermediate increase in Conc II, suggesting a negative association with the $\mathrm{pH}$ trend (Kröger et al., 2017). According to previous research, there is no clear increasing or decreasing trend for butyrate during concentrate feeding and lowered $\mathrm{pH}$ conditions (Kleen et al., 2003; Wang et al., 2013; Danscher et al., 2015).
Bacteria traditionally known to be butyrate producers, including Butyrivibrio spp., Pseudobutyrivibrio spp., and Coprococcus spp. (van Gylswyk et al., 1996), were decreased with concentrate feeding. However, several other bacteria that are also able to produce butyrate, increased during concentrate feeding, such as Roseburia spp. (van Gylswyk et al., 1996), Megasphaera spp. (Puniya et al., 2015), and Pseudoramibacter spp. (Whitman, 2009). The literature also shows no consistent trend for rumen butyrate producers, such as Butyrivibrio spp. and Pseudobutyrivibrio spp., with concentrate feeding (Hook et al., 2011; Petri et al., 2013; McCann et al., 2016). This inconsistent response of butyrate producers to concentrate feeding was also reflected in our finding that the only taxon that showed differing trends for Conc I and Con II in comparison to RD was a Butyrivibrio fibrisolvens strain (OTU_62). Further research is required to look at the functional overlap of various bacterial taxa, as well as their interaction with protozoa, fungi, archaea, and the host, to determine not only their metabolic capabilities but also their metabolic contributions to fermentation in the rumen (Firkins and Yu, 2015).

\section{Effects of Feed Additive Supplementation}

The second aim of our study was to determine the effects of PHY or AY supplementation on rumen fermentation and bacterial changes when cows are challenged intermittently with high-concentrate diets. Overall, most of the effects of the feed additives on the rumen bacterial community occurred during the first concentrate challenge, which was expected based on decreased pH in Conc I (Kröger et al., 2017), and

Table 1. Influence of phytogenic (PHY) and autolyzed yeast (AY) on short-chain fatty acid (SCFA) profile, lactate, and ammonia in the particle-associated liquid during 2 high-concentrate feeding phases ${ }^{1}$

\begin{tabular}{|c|c|c|c|c|c|c|c|c|c|c|}
\hline \multirow[b]{2}{*}{ Parameter } & \multicolumn{3}{|c|}{ Conc I } & \multirow[b]{2}{*}{ SEM } & \multirow[b]{2}{*}{$P$-value } & \multicolumn{3}{|c|}{ Conc II } & \multirow[b]{2}{*}{ SEM } & \multirow[b]{2}{*}{$P$-value } \\
\hline & $\mathrm{CON}$ & PHY & AY & & & $\mathrm{CON}$ & PHY & AY & & \\
\hline Acetate & 54.7 & 55.9 & 55.3 & 1.10 & 0.35 & $53.8^{\mathrm{a}}$ & $53.8^{\mathrm{a}}$ & $50.8^{\mathrm{b}}$ & 1.11 & 0.03 \\
\hline Propionate & $23.8^{(\mathrm{a})}$ & $21.0^{(\mathrm{b})}$ & $23.3^{(\mathrm{a})}$ & 1.16 & 0.09 & 27.5 & 25.1 & 29.9 & 1.18 & 0.14 \\
\hline Butyrate & $13.0^{\mathrm{b}}$ & $16.0^{\mathrm{a}}$ & $14.0^{\mathrm{ab}}$ & 0.89 & 0.01 & $11.8^{(\mathrm{b})}$ & $13.9^{\text {(a) }}$ & $11.9^{(\mathrm{ab})}$ & 0.89 & 0.08 \\
\hline Iso-valerate & $0.8^{(\mathrm{b})}$ & $1.2^{(\mathrm{a})}$ & $1.0^{(\mathrm{ab})}$ & 0.16 & 0.08 & $1.1^{\mathrm{a}}$ & $1.3^{\mathrm{a}}$ & $0.6^{\mathrm{b}}$ & 0.16 & 0.01 \\
\hline Iso-butyrate & 0.8 & 0.8 & 0.7 & 0.05 & 0.62 & 0.7 & 0.8 & 0.6 & 0.05 & 0.12 \\
\hline Acetate:propionate & 2.7 & 2.8 & 2.6 & 0.22 & 0.65 & 2.0 & 2.4 & 1.7 & 0.22 & 0.23 \\
\hline Total lactate $(\mathrm{m} M)$ & $1.01^{(\mathrm{b})}$ & $0.74^{(\mathrm{b})}$ & $2.89^{(\mathrm{a})}$ & 0.85 & 0.09 & 1.99 & 2.07 & 1.87 & 0.852 & 0.91 \\
\hline D-lactate $(\mathrm{m} M)$ & 0.40 & 0.34 & 1.19 & 0.42 & 0.16 & 0.89 & 1.09 & 0.78 & 0.42 & 0.72 \\
\hline L-lactate $(\mathrm{m} M)$ & $0.61^{(\mathrm{b})}$ & $0.40^{(\mathrm{b})}$ & $1.70^{(\mathrm{a})}$ & 0.44 & 0.06 & 1.10 & 0.98 & 1.09 & 0.44 & 0.83 \\
\hline Ammonia $(\mathrm{m} M)$ & 2.37 & 1.77 & 1.86 & 0.54 & 0.34 & 1.24 & 2.11 & 1.30 & 0.542 & 0.17 \\
\hline
\end{tabular}

$\overline{\mathrm{a}, \mathrm{b}}$ Different superscript letters indicate significant $(P<0.05)$ changes or a trend (a,b in parentheses; $0.05<P<0.1)$ between the feed additive groups (PHY, AY) compared with the control group (CON).

${ }^{1}$ Conc $\mathrm{I}=65 \%$ concentrate for $1 \mathrm{wk}$; Conc II $=65 \%$ concentrate for $2 \mathrm{wk}$. 


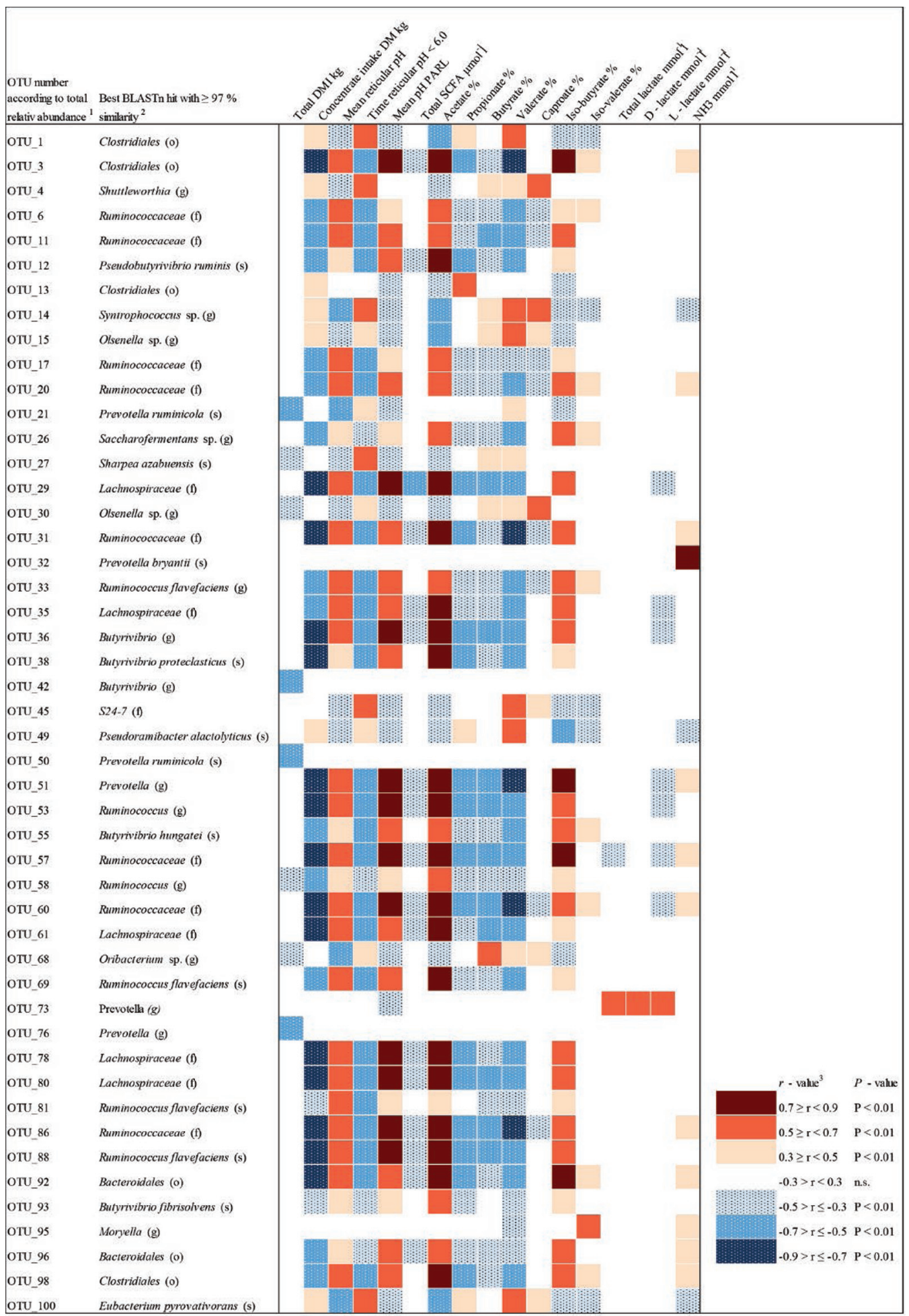

Figure 7. Pearson correlation analyses of the most abundant 100 operational taxonomic units (OTU) with rumen fermentation relevant parameters. Only OTU that showed at least a moderate correlation to one of the parameters are shown in this figure. Taxonomic assignments based on the higher sequence identity to the reference database, and either the result from OTU picking against SILVA (https://www.arb-silva. de, version 123; last accessed August 25, 2016) or the best Basic Local Alignment Search Tool nucleotide (BLASTn) hit against National Center for Biotechnology Information was chosen as the taxonomic assignment. The letter in parentheses is indicative of the taxonomic level reached with at least $97 \%$ identity: order (o), family (f), genus (g), and species (s). Pearson correlation coefficient $\mathrm{r}$ values $|<0.3|$ are considered as negligible, $|0.3 \leq \mathrm{r}<0.5|$ as low, $|0.5 \leq \mathrm{r}<0.7|$ as moderate, $|0.7 \leq \mathrm{r}<0.9|$ as high; n.s. $=$ not significant $(P>0.1)$. SCFA $=$ short-chain fatty acid; PARL $=$ particle-associated rumen liquid. Color version available online. 
especially because PHY has been previously described as effective under low $\mathrm{pH}$ conditions (Cardozo et al., 2005). Almost all of the bacteria that were decreased by PHY are known starch utilizers, including the genera Shuttleworthia, Olsenella, Bacteroides, Bifidobacterium, Roseburia, and Syntrophococcus (Calsamiglia et al., 2007; Patra, 2011), whereas no fiber fermenting taxa were reduced by PHY. Previous research has indicated that PHY has a bacteriostatic effect affecting cell wall electrolyte exchange, which diverts the cell from metabolic processes (Calsamiglia et al., 2007; Cobellis et al., 2016). In a high-concentrate diet, the increase in starch provided would normally supply amylolytic bacteria with sufficient substrate to promote growth. The decrease of amylolytic bacteria, seen with supplementation of $\mathrm{PHY}$ in this experiment, supports the mode of action stated by Calsamiglia et al. (2007) and Cobellis et al. (2016). A reduction in starch utilizers would potentially delay the onset of SCFA fermentation, reduce SCFA accumulation, and thereby prevent rapid and extended duration of low $\mathrm{pH}$ after feeding. Moreover, with a decrease in starch utilizers, cellulolytic bacteria might have a chance to evolve due to less competition (Patra and $\mathrm{Yu}, 2015$ ). This is supported by the higher reticulo-rumen $\mathrm{pH}$ previously reported for PHY (Kröger et al., 2017). A distinct effect on certain bacterial groups according to Gram staining was seen, where all GNB decreased and almost all GPB increased for both feed additives. These effects of the feed additive should be further investigated, as some GNB species harbor potential LPS producers and GPB, among others, some major cellulolytic bacteria. However, in vitro studies have suggested that GNB show a higher resistance than GPB against different plant-derived substances because their outer membrane is hydrophilic and does not allow the entrance of lipophilic substances (Cardozo et al., 2005; Calsamiglia et al., 2007; Hart et al., 2008). Thus, our in vivo data produced in cattle fed concentrate-rich diets suggests another potential mode of action for plant-derived substances, which requires more research to be elucidated. For the AY group, the effects on both GNB and GPB are in agreement with literature. As effective ingredient, certain yeastcell wall components such as mannan-oligosaccharides, $\beta$-glucans, chitin, peptides, AA, and nucleotides can act as high-affinity ligands for GNB and remove them from the digestive system without attachment and colonization (Nocek et al., 2011; Ganner and Schatzmayr, 2012). Accordingly, AY decreased Bacteroides, a major gram-negative starch fermenting and ammonia utilizing genus (Dworkin, 2006b), during Conc I. Moreover, cell wall components found in AY have also been found to act as substrate for cellulolytic bacteria, promoting growth in the challenged rumen (Harrison et al.,
1988). This suggested prebiotic effect was reflected in our finding that AY increased both Ruminococcus spp. significantly and Clostridium spp. by trend, throughout the experiment, both of which are known to represent a large number of cellulose degrading rumen species (Whitman, 2009). The increase in cellulolytic bacteria, despite high-concentrate feeding and the reduction of starch fermenting GNB by AY, would contribute to the maintenance of a physiological ruminal $\mathrm{pH}$, which was shown by our accompanying study, where AY tended to stabilize reticulo-ruminal $\mathrm{pH}$ despite higher DMI (Kröger et al., 2017).

We reported that with lower $\mathrm{pH}$, butyrate increased in this feeding model; however, PHY increased both $\mathrm{pH}$ and butyrate in Conc I. Therefore, it remains to be clarified if PHY has a direct effect on microbial butyrate production and metabolism. Nevertheless, an increased butyrate level with PHY could have favored epithelial uptake of other metabolites and so positively affected ruminal $\mathrm{pH}$ because it was described that butyrate is a trigger for enhanced papillae growth and absorption capacity (Steele et al., 2011; Baldwin et al., 2012). In line with other studies (Mao et al., 2013a; Li et al., 2016), the application of AY corresponded with decreased acetate in Conc II, suggesting that AY can also influence the metabolism of cellulolytic bacteria.

\section{CONCLUSIONS}

High-concentrate feeding clearly decreased rumen bacterial diversity, with alleviated effects in the second concentrate challenge, revealing a possible adaptation mechanism of rumen bacteria. Interestingly, some GNB were increased with concentrate feeding and low $\mathrm{pH}$, which suggests reevaluation of the $\mathrm{pH}$ sensitivity and substrate usage of their species. Our findings suggest that both the PHY and AY feed additive evolve their effects on the rumen microbiome predominantly during low ruminal $\mathrm{pH}$ conditions. An interesting finding was that both PHY and AY decreased GNB and increased GPB, which provides background for further research on the mode of action of those 2 feed additives. Autolyzed yeast provided the opportunity to increase cellulolytic bacteria and decrease certain starch fermenters, whereas PHY showed a decreasing effect on overall bacterial diversity and starch fermenting bacteria and increased butyrate levels, which could have contributed to the higher and stabilized reticulo-ruminal $\mathrm{pH}$ levels during this experiment.

\section{ACKNOWLEDGMENTS}

This research is part of the K-project "ADDA-Advancement of Dairying in Austria" funded by the Aus- 
trian Research Promotion Agency (FFG; Vienna, Austria) and Biomin Holding GmbH (Tulln, Austria) being part of this project under the scope of the Competence Centers for Excellent Technologies (COMET) program (grant no. 843543). Partial funding for this project was also provided by the University of Veterinary Medicine (Vienna) postdoctoral program. Special thanks go to the staff of the teaching and research farm Kremesberg (Pottensteinl, Austria) for the help during the feeding experiment, the laboratory team at the Institute for Animal Nutrition and Functional Plant Compounds (Vetmeduni, Vienna, Austria) for the laboratory work, and Königshofer Futtermittel Assmannmühlen GmbH (Ebergassing, Austria) for providing the concentrate feed. Further thanks go to Monika Dzieciol and Stefanie Wetzels (Institute of Milk Hygiene, Milk Technology and Food Science, Vetmeduni, Vienna, Austria) for support during bioinformatic analysis.

\section{REFERENCES}

Aschenbach, J. R., G. B. Penner, F. Stumpff, and G. Gäbel. 2011 Ruminant nutrition symposium: Role of fermentation acid absorption in the regulation of ruminal pH. J. Anim. Sci. 89:1092-1107. https://doi.org/10.2527/jas.2010-3301.

Baldwin, R. L., VI, S. Wu, W. Li, C. Li, B. J. Bequette, and R. W. Li. 2012. Quantification of transcriptome responses of the rumen epithelium to butyrate infusion using RNA-seq technology. Gene Regul. Syst. Bio. 6:67-80. https://doi.org/10.4137/GRSB.S9687.

Calsamiglia, S., M. Busquet, P. W. Cardozo, L. Castillejos, and A Ferret. 2007. Invited review: Essential oils as modifiers of rumen microbial fermentation. J. Dairy Sci. 90:2580-2595. https://doi .org/10.3168/jds.2006-644.

Cardozo, P. W., S. Calsamiglia, A. Ferret, and C. Kamel. 2005. Screening for the effects of natural plant extracts at different $\mathrm{pH}$ on in vitro rumen microbial fermentation of a high-concentrate diet for beef cattle. J. Anim. Sci. 83:2572-2579.

Chen, S., L. Niu, and Y. Zhang. 2010. Saccharofermentans acetigenes gen. nov., sp. nov., an anaerobic bacterium isolated from sludge treating brewery wastewater. Int. J. Syst. Evol. Microbiol. 60:2735-2738. https://doi.org/10.1099/ijs.0.017590-0.

Cobellis, G., M. Trabalza-Marinucci, and Z. Yu. 2016. Critical evaluation of essential oils as rumen modifiers in ruminant nutrition: A review. Sci. Total Environ. 545-546:556-568. https://doi.org/10 .1016/j.scitotenv.2015.12.103.

Danscher, A. M., S. Li, P. H. Andersen, E. Khafipour, N. B. Kristensen, and J. C. Plaizier. 2015. Indicators of induced subacute ruminal acidosis (SARA) in Danish Holstein cows. Acta Vet. Scand. 57:39. https://doi.org/10.1186/s13028-015-0128-9.

Dewhirst, F. E., B. J. Paster, N. Tzellas, B. Coleman, J. Downes, D. A. Spratt, and W. G. Wade. 2001. Characterization of novel human oral isolates and cloned $16 \mathrm{~S}$ rDNA sequences that fall in the family Coriobacteriaceae: Description of Olsenella gen. nov. reclassification of Lactobacillus uli as Olsenella uli comb. nov. and description of Olsenella profusa sp. nov. Int. J. Syst. Evol. Microbiol. 51:1797-1804. https://doi.org/10.1099/00207713-51-5-1797.

Dijkstra, J., J. L. Ellis, E. Kebreab, A. B. Strathe, S. López, J. France, and A. Bannink. 2012. Ruminal pH regulation and nutritional consequences of low pH. Anim. Feed Sci. Technol. 172:22-33. https:// doi.org/10.1016/j.anifeedsci.2011.12.005.

Dworkin, M. 2006a. The Prokaryotes Vol 3: Archaea. Bacteria: Firmicutes, Actinomycetes. 3rd ed. M. Dworkin, S. Falkow, E. Rosenberg, K.-H. Schleifer, and E. Stackebrandt, ed. Springer, New York, NY.
Dworkin, M. 2006b. The Prokaryotes Vol 7: Protebacteria: Delta and Epsilon Subclasses. Deeply Rooting Bacteria. 3rd ed. M. Dworkin, S. Falkow, E. Rosenberg, K.-H. Schleifer, and E. Stackebrandt, editors. Springer, New York, NY.

Firkins, J. L., and Z. Yu. 2015. Ruminant nutrition symposium: How to use data on the rumen microbiome to improve our understanding of ruminant nutrition. J. Anim. Sci. 93:1450-1470. https://doi .org/10.2527/jas.2014-8754.

Ganner, A., and G. Schatzmayr. 2012. Capability of yeast derivatives to adhere enteropathogenic bacteria and to modulate cells of the innate immune system. Appl. Microbiol. Biotechnol. 95:289-297. https://doi.org/10.1007/s00253-012-4140-y.

Harrison, G. A., R. W. Hemken, K. A. Dawson, R. J. Harmon, and K. B. Barker. 1988. Influence of addition of yeast culture supplement to diets of lactating cows on ruminal fermentation and microbial populations. J. Dairy Sci. 71:2967-2975. https://doi.org/10.3168/ jds.S0022-0302(88)79894-X

Hart, K. J., D. R. Yáñez-Ruiz, S. M. Duval, N. R. McEwan, and C. J. Newbold. 2008. Plant extracts to manipulate rumen fermentation. Anim. Feed Sci. Technol. 147:8-35. https://doi.org/10.1016/ j.anifeedsci.2007.09.007.

Henderson, G., F. Cox, S. Ganesh, A. Jonker, W. Young, and P. H. Janssen. 2015. Rumen microbial community composition varies with diet and host, but a core microbiome is found across a wide geographical range. Sci. Rep. 5:14567. https://doi.org/10.1038/ srep14567.

Hinkle, D. E., W. Wiersma, and S. G. Jurs. 2003. Applied statistics for the behavioral sciences. 5th ed. Houghton Mifflin, Boston, MA

Hook, S. E. M. A. Steele, K. S. Northwood, J. Dijkstra, J. France, A. D. G. Wright, and B. W. McBride. 2011. Impact of subacute ruminal acidosis (SARA) adaptation and recovery on the density and diversity of bacteria in the rumen of dairy cows. FEMS Microbiol. Ecol. 78:275-284. https://doi.org/10.1111/j.1574-6941.2011 $.01154 . \mathrm{x}$

Kesselring, J., C. Stoiber, I. Schantl, and G. Schatzmayr. 2017. Autolyzed yeast supplementation specifically alters the microbial diversity in fibrous rumen content in vitro. In 16.Boku-Symposium Tierernährung - Der gesunde Verdauungstrakt. Vienna, Austria. 28.

Khafipour, E., S. Li, J. C. Plaizier, and D. O. Krause. 2009. Rumen microbiome composition determined using two nutritional models of subacute ruminal acidosis. Appl. Environ. Microbiol. 75:71157124. https://doi.org/10.1128/AEM.00739-09.

Kleen, J. L., G. A. Hooijer, J. Rehage, and J. P. T. M. Noordhuizen. 2003. Subacute ruminal acidosis (SARA): A review. J. Vet. Med. A Physiol. Pathol. Clin. Med. 50:406-414. https://doi.org/10.1046/j .1439-0442.2003.00569.x.

Kong, Y., R. Teather, and R. Forster. 2010. Composition, spatial distribution, and diversity of the bacterial communities in the rumen of cows fed different forages. FEMS Microbiol. Ecol. 74:612-622. https://doi.org/10.1111/j.1574-6941.2010.00977.x.

Kröger, I., E. Humer, V. Neubauer, N. Reisinger, S. Aditya, and Q. Zebeli. 2017. Modulation of chewing behavior and reticular $\mathrm{pH}$ in non-lactating cows challenged with concentrate-rich diets supplemented with phytogenic compounds and autolyzed yeast. J. Dairy Sci. 100:9702-9714. https://doi.org/10.3168/jds.2017-12755.

Li, S., I. Yoon, M. Scott, E. Khafipour, and J. C. Plaizier. 2016. Impact of Saccharomyces cerevisiae fermentation product and subacute ruminal acidosis on production, inflammation, and fermentation in the rumen and hindgut of dairy cows. Anim. Feed Sci. Technol. 211:50-60. https://doi.org/10.1016/j.anifeedsci.2015.10.010.

Liu, C., S. M. Finegold, Y. Song, and P. A. Lawson. 2008. Reclassification of Clostridium coccoides, Ruminococcus hansenii, Ruminococcus hydrogenotrophicus, Ruminococcus luti, Ruminococcus productus and Ruminococcus schinkii as Blautia coccoides gen. nov., comb. nov., Blautia hansenii comb. nov., Blautia hydrogenotrophica comb. nov., Blautia luti comb. nov., Blautia producta comb. nov., Blautia schinkii comb. nov. and description of Blautia wexlerae sp. nov., isolated from human faeces. Int. J. Syst. Evol. Microbiol. 58:1896-1902. https://doi.org/10.1099/ijs.0.65208-0. 
Lozupone, C., and R. Knight. 2005. UniFrac: A new phylogenetic method for comparing microbial communities. Appl. Environ. Microbiol. 71:8228-8235. https://doi.org/10.1128/AEM.71.12.8228 -8235.2005 .

Mao, H.-L., H.-L. Mao, J. K. Wang, J. X. Liu, and I. Yoon. 2013a. Effects of Saccharomyces cerevisiae fermentation product on in vitro fermentation and microbial communities of low-quality forages and mixed diets. J. Anim. Sci. 91:3291-3298. https://doi.org/10.2527/ jas.2012-5851.

Mao, S. Y., R. Y. Zhang, D. S. Wang, and W. Y. Zhu. 2013b. Impact of subacute ruminal acidosis (SARA) adaptation on rumen microbiota in dairy cattle using pyrosequencing. Anaerobe 24:12-19. https://doi.org/10.1016/j.anaerobe.2013.08.003.

McCann, J. C., S. Luan, F. C. Cardoso, H. Derakhshani, E. Khafipour, and J. J. Loor. 2016. Induction of subacute ruminal acidosis affects the ruminal microbiome and epithelium. Front. Microbiol. 7:701 https://doi.org/10.3389/fmicb.2016.00701.

Mukherjee, S., D. Stamatis, J. Bertsch, G. Ovchinnikova, O. Verezemska, M. Isbandi, A. D. Thomas, R. Ali, K. Sharma, N. C. Kyrpides, and T. B. K. Reddy. 2017. Genomes OnLine Database (GOLD) v.6: data updates and feature enhancements. Nucleic Acids Res. 45:D446-D456. https://doi.org/10.1093/nar/gkw992.

Nagaraja, T. G., and E. C. Titgemeyer. 2007. Ruminal acidosis in beef cattle: The current microbiological and nutritional outlook. J. Dairy Sci. 90:E17-E38. https://doi.org/10.3168/jds.2006-478.

Navas-Molina, J. A., J. M. Peralta-Sánchez, A. González, P. J. McMurdie, Y. Vázquez-Baeza, Z. Xu, L. K. Ursell, C. Lauber, H. Zhou, S. Jin Song, J. Huntley, G. Ackermann, D. Berg-Lyons, S. Holmes, J. G. Caporaso, and R. Knight. 2013. Advancing our understanding of the human microbiome using QIIME. 531. 371-444 pp.

Nocek, J. E., M. G. Holt, and J. Oppy. 2011. Effects of supplementation with yeast culture and enzymatically hydrolyzed yeast on performance of early lactation dairy cattle. J. Dairy Sci. 94:40464056. https://doi.org/10.3168/jds.2011-4277.

Oliveros, J. C. 2007. Venny. An interactive tool for comparing lists with Venn's diagrams. Accessed May 17, 2017. http://bioinfogp .cnb.csic.es/tools/venny/index.html.

Patra, A. K. 2011. Effects of essential oils on rumen fermentation, microbial ecology and ruminant production. Asian J. Anim. Vet. Adv. 6:416-428. https://doi.org/10.3923/ajava.2011.416.428.

Patra, A. K., and Z. Yu. 2015. Essential oils affect populations of some rumen bacteria in vitro as revealed by microarray (RumenBactArray) analysis. Front. Microbiol. 6:297. https://doi.org/10.3389/ fmicb.2015.00297.

Peterson, J., S. Garges, M. Giovanni, P. McInnes, L. Wang, J. Schloss, V. Bonazzi, J. McEwen, K. Wetterstrand, C. Deal, C. Baker, V. Di Francesco, T. Howcroft, R. Karp, R. Lunsford, C. Wellington, T. Belachew, M. Wright, C. Giblin, H. M. David, and M. Guyer. 2009. The NIH human microbiome project. Genome Res. 19:2317-2323. https://doi.org/10.1101/gr.096651.109.

Petri, R. M., T. Schwaiger, G. B. Penner, K. A. Beauchemin, R. J. Forster, J. J. McKinnon, and T. A. McAllister. 2013. Characterization of the core rumen microbiome in cattle during transition from forage to concentrate as well as during and after an acidotic challenge. PLoS One 8:e83424. https://doi.org/10.1371/journal .pone.0083424.

Plaizier, J. C., E. Khafipour, S. Li, G. N. Gozho, and D. O. Krause. 2012. Subacute ruminal acidosis (SARA), endotoxins and health consequences. Anim. Feed Sci. Technol. 172:9-21. https://doi.org/ 10.1016/j.anifeedsci.2011.12.004.

Pourazad, P., R. Khiaosa-ard, M. Qumar, S. U. Wetzels, F. Klevenhusen, B. U. Metzler-Zebeli, and Q. Zebeli. 2016. Transient feeding of a concentrate-rich diet increases the severity of subacute ruminal acidosis in dairy cattle. J. Anim. Sci. 94:726-738. https://doi.org/ $10.2527 /$ jas.2015-9605.

Puniya, A. K., R. Singh, and D. N. Kamra. 2015. Rumen Microbiology: From Evolution to Revolution. 1st ed. Springer India, New Delhi.

Purushe, J., D. E. Fouts, M. Morrison, B. A. White, R. I. Mackie, P. M. Coutinho, B. Henrissat, and K. E. Nelson. 2010. Comparative genome analysis of Prevotella ruminicola and Prevotella bryantii: Insights into their environmental niche. Microb. Ecol. 60:721-729. https://doi.org/10.1007/s00248-010-9692-8.

Qumar, M., R. Khiaosa-ard, P. Pourazad, S. U. Wetzels, F. Klevenhusen, W. Kandler, J. R. Aschenbach, and Q. Zebeli. 2016. Evidence of in vivo absorption of lactate and modulation of short chain fatty acid absorption from the reticulorumen of non-lactating cattle fed high concentrate diets. PLoS One 11:e0164192. https://doi.org/10 .1371/journal.pone.0164192.

Steele, M. A., J. Croom, M. Kahler, O. AlZahal, S. E. Hook, K. Plaizier, and B.W. McBride. 2011. Bovine rumen epithelium undergoes rapid structural adaptations during grain-induced subacute ruminal acidosis. Am. J. Physiol. Regul. Integr. Comp. Physiol. 300:R1515-R1523. https://doi.org/10.1152/ajpregu.00120.2010.

Stevenson, D. M., and P. J. Weimer. 2007. Dominance of Prevotella and low abundance of classical ruminal bacterial species in the bovine rumen revealed by relative quantification real-time PCR. Appl. Microbiol. Biotechnol. 75:165-174. https://doi.org/10.1007/ s00253-006-0802-y.

van Gylswyk, N. O., H. Hippe, and F. A. Rainey. 1996. Pseudobutyrivibrio ruminis gen. nov., sp. nov., a butyrate-producing bacterium from the rumen that closely resembles Butyrivibrio fibrisolvens in phenotype. Int. J. Syst. Bacteriol. 46:559-563. https://doi.org/10 .1099/00207713-46-2-559.

van Houtert, M. F. J. 1993. The production and metabolism of volatile fatty acids by ruminants fed roughages: A review. Anim. Feed Sci. Technol. 43:189-225. https://doi.org/10.1016/0377-8401(93)90078 $-\mathrm{X}$.

Wang, D. S., R. Y. Zhang, W. Y. Zhu, and S. Y. Mao. 2013. Effects of subacute ruminal acidosis challenges on fermentation and biogenic amines in the rumen of dairy cows. Livest. Sci. 155:262-272. https://doi.org/10.1016/j.livsci.2013.05.026.

Weatherburn, M. W. 1967. Phenol-hypochlorite reaction for determination of ammonia. Anal. Chem. 39:971-974.

Whitman, W. B. 2009. Bergey's Manual of Systematic Bacteriology. Vol. 3: The Firmicutes. 2nd ed. P. De Vos, G. M. Garrity, D. Jones, N. R. Krieg, W. Ludwig, F. A. Rainey, K.-H. Schleifer, and W. B. Whitman, ed. Springer, New York, NY.

Zebeli, Q., M. Tafaj, B. Junck, D. Mansmann, H. Steingass, and W. Drochner. 2008. Effects of dietary forage particle size and concentrate level on fermentation profile, in vitro degradation characteristics and concentration of liquid- or solid-associated bacterial mass in the rumen of dairy cows. Arch. Anim. Nutr. 62:230-240. https://doi.org/10.1080/17450390802027486. 